\title{
Constitutive Expression and Enzymatic Cleavage of ICAM-1 in the Spontaneously Hypertensive Rat
}

\author{
Sheng Tong ${ }^{a}$ Hanmanth J. Neboori ${ }^{b}$ Edward D. Tran ${ }^{b}$ \\ Geert W. Schmid-Schönbein ${ }^{b}$ \\ aWallace H. Coulter Department of Biomedical Engineering, Georgia Institute of Technology, Atlanta, Ga., and \\ ${ }^{b}$ Department of Bioengineering, The Whitaker Institute of Biomedical Engineering, University of California \\ San Diego, La Jolla, Calif., USA
}

\section{Key Words}

Leukocyte adhesion • Endothelium • Receptor cleavage •

Arterioles $\cdot$ Venules

\begin{abstract}
Background/Aims: Leukocyte adhesion to the endothelium is abnormal in hypertension. We have recently shown that spontaneously hypertensive rats (SHRs) have circulating leukocytes with enhanced $\mathrm{CD} 18$ receptor cleavage. In the current study, we investigate expression levels of its counter receptor, intercellular adhesion molecule (ICAM-1), and its possible proteolytic cleavage in the SHR and control Wistar rat. Methods: ICAM-1 was labeled on tissue sections with two antibodies targeting its extracellular and intracellular domains and evaluated by light absorption measurements. The in situ cleavage of ICAM-1 was assessed by treating vessel sections with matrix metalloproteinase (MMP)-7, MMP-9 and elastase. Results: SHRs showed a significant increase in ICAM-1 expression in liver and kidney compared with Wistar rats. The liver and kidney glomeruli exhibit a discrepancy in label density between intra- and extracellular antibodies, which suggests that enzymatic cleavage may be a factor determining ICAM-1 distribution. MMP-7 and MMP-9, which are
\end{abstract}

elevated in SHR plasma, and elastase, which has elevated activity in SHR neutrophils, cleave the extracellular domain of ICAM-1 when applied to the tissue. Conclusion: ICAM-1 expression in SHRs is upregulated in a tissue-specific manner. Proteolytic cleavage of the extracellular domain of ICAM-1 and accumulation in kidney glomeruli may play a role in the renal involvement of inflammation.

Copyright $\odot 2011$ S. Karger AG, Basel

\section{Introduction}

Organ damage associated with primary hypertension may be accompanied by a prolonged inflammatory state, including infiltration of monocytes/macrophages and lymphocytes into the tissues [1-5], but little is known about the molecular mechanisms governing leukocyte infiltration in hypertension. Leukocyte migration across the endothelium and into surrounding tissue depends on the interaction between selectins, integrins and their corresponding ligands on the membrane of vascular endothelium and leukocytes [6]. Suematsu et al. [7] found that leukocyte adhesion was attenuated in the mesenteric microcirculation of spontaneously hypertensive rats (SHRs)

\section{KARGER}

(C) 2011 S. Karger AG, Basel

Fax +41613061234

E-Mail karger@karger.ch

www.karger.com
Accessible online at:

www.karger.com/jvr
Dr. Geert W. Schmid-Schönbein

Department of Bioengineering, The Whitaker Institute of Biomedical Engineering

University of California San Diego

La Jolla, CA 92093-0412 (USA)

Tel. +1 858534 3852, E-Mail gwss@ @ioeng.ucsd.edu 
Table 1. Physiological parameters

\begin{tabular}{llll}
\hline Rat strain & $\begin{array}{l}\text { Age } \\
\text { days }\end{array}$ & $\begin{array}{l}\text { Body } \\
\text { weight, g }\end{array}$ & $\begin{array}{l}\text { Mean blood } \\
\text { pressure, mm Hg }\end{array}$ \\
\hline Wistar & $132 \pm 19$ & $469 \pm 30$ & $96 \pm 8$ \\
SHR & $130 \pm 9$ & $336 \pm 16^{*}$ & $140 \pm 14^{*}$ \\
\hline
\end{tabular}

${ }^{*} \mathrm{p}<0.05$ compared to Wistar strain.

compared with normotensive Wistar Kyoto rats due to impaired P-selectin expression. SHR postcapillaries have a reduced expression level of P-selectin [8] and SHR neutrophils have reduced CD18 levels [9]. Few other adhesion molecules that contribute to leukocyte infiltration, especially in organs susceptible to damage in hypertension, have been investigated.

Intercellular adhesion molecule-1 (ICAM-1), a CD18 receptor, mediates firm leukocyte adhesion to endothelium, transendothelial migration and attachment to parenchymal cells [6]. ICAM-1 is constitutively expressed on vascular endothelial cells in postcapillary venules, and is upregulated during inflammation, in response to proinflammatory agents [6] or upon its exposure after cleavage of the glycocalyx [10]. ICAM-1 is a key mediator of leukocyte recruitment in various diseases involving leukocyte-induced tissue injuries [11-13]. Likewise, SHR neutrophils can damage activated endothelial cells expressing ICAM-1, and the damage is alleviated by inhibiting ICAM-1-mediated cell adhesion [14]. Antihypertensive drugs such as amlodipine and hydralazine have been shown to reduce leukocyte migration in SHRs, which is accompanied by decreased ICAM-1 expression on vascular endothelium $[15,16]$. Moreover, C-reactive protein, angiotensin II and oxygen free radicals, which play important roles in the pathology of hypertension, are able to induce ICAM-1 expression through NF- $\mathrm{kB}$-dependent pathways [17, 18].

Evaluation of constitutive ICAM-1 expression on vascular endothelium at the organ level shows that compared to Wistar Kyoto rats, ICAM-1 expression is increased in the stomach but not in the heart, skeletal muscle and brain of SHRs [19]. It should be noted that ICAM-1 expression is heterogeneous on vascular endothelium [20]. Increased leukocyte infiltration may result from upregulation of ICAM-1 in selected microvascular regions, which may not be detectable with a whole-organ approach. Several clinical studies have shown an increase in soluble ICAM-1 in the plasma of hypertensive patients
$[21,22]$. Soluble ICAM-1 can be formed by proteolytic cleavage of the extracellular domain of the membranebound ICAM-1 by elastase, cathepsin G and matrix metalloproteinase-9 (MMP-9) [23-26]. Yasmin et al. [27] found that the plasma activities of elastase and MMP-9 were increased in patients with systolic hypertension. We have recently shown that SHR plasma has enhanced MMP activity (including MMP-7, MMP-9) [28] and that the reduced CD18 levels in SHRs are in fact due to proteolytic cleavage of the extracellular domain of CD18 by MMPs [29]. Therefore, we hypothesize that proteolytic cleavage may also influence extracellular domain levels of ICAM-1 in hypertension.

Thus, our aim was to elucidate ICAM-1 expression at the microvascular level in heart, skeletal muscle, liver and kidney. ICAM-1 in selected tissues of SHRs and normotensive Wistar rats was measured by immunohistochemistry in combination with light absorption measurements on tissue sections. To investigate whether there is proteolytic cleavage of ICAM-1, we compared immunolabeling by two different antibodies that separately targeted the intracellular and extracellular domains of ICAM-1. In addition, we studied the direct effect of MMP-7, MMP-9 and elastase on ICAM-1 expression.

\section{Materials and Methods}

\section{Materials}

ICAM-1 antibodies (clone 1A29, mouse monoclonal IgG targeting ICAM-1 extracellular domain [19] and clone M-19, goat polyclonal IgG targeting ICAM-1 intracellular domain [30]), control mouse IgG and control goat IgG were purchased from Santa Cruz. Biotinylated secondary antibodies (horse anti-mouse IgG, rat absorbed, and horse anti-goat IgG), biotin- and avidin-blocking solutions, Vectastain Elite ABC kit and Vector NovaRed horseradish peroxidase substrate were purchased from Vector Laboratories. Porcine pancreatic elastase type IV, N-succinylAla-Ala-Ala $p$-nitroanilide (SucAla 3 -PNA), and N-methoxysuccinyl-Ala-Ala-Pro-Val-7-amido-4-methylcoumarin (AAPVmethylcoumarin) were purchased from Sigma-Aldrich. Quantikine rat sICAM-1 immunoassay was purchased from R\&D systems. Recombinant human MMP-7 and MMP-9 were purchased from Calbiochem.

\section{Animal Procedures}

Age-matched male SHRs and Wistar rats (15-20 weeks, Charles River Laboratories) (table 1) were anesthetized by intraperitoneal injection of sodium pentobarbital $(50 \mathrm{mg} / \mathrm{kg}$ body weight). The femoral artery was cannulated (PE 50 tubing) and after recording of arterial blood pressure, an aliquot of blood $(2 \mathrm{ml})$ was withdrawn directly into a syringe containing heparin solution (20 units/ml blood). The heparinized blood samples were immediately centrifuged at 5,000 $\mathrm{g}$ for $10 \mathrm{~min}$ at room temperature. The plasma was collected and stored at $-80^{\circ} \mathrm{C}$ until further 
analysis. The circulation was perfused with phosphate-buffered saline (PBS) and organs of interest were harvested and frozen in liquid nitrogen. All animal procedures were reviewed and approved by the University of California San Diego Animal Subjects Committee.

\section{Immunohistochemistry}

ICAM-1 levels were examined with an antibody that targets the extracellular domain (1A29) and another for the intracellular domain (M-19) of ICAM-1. In brief, frozen tissue blocks were cut into $8-\mu \mathrm{m}$ sections. The tissue sections were fixed in cold acetone and air-dried. Six sections from each organ were equally divided into two groups for labeling the extracellular or intracellular domains of ICAM-1. Tissue sections from the SHRs and Wistar rats were paired during labeling in order to minimize the labeling variation between the two groups. The sections were hydrated with PBS and sequentially treated with $0.6 \%$ hydrogen peroxide, avidin solution, biotin solution and $5 \%$ normal horse serum to block nonspecific labeling. Thereafter, the sections were incubated with one of the anti-ICAM-1 antibodies (1:200 dilution in 5\% normal horse serum) followed by a biotinylated secondary antibody. The Vectastain Elite ABC kit and Vector NovaRed horseradish peroxidase substrate were applied for antigen visualization. Negative controls were performed for each group by replacing the primary antibody with control mouse/goat IgG.

\section{Image Acquisition and Analysis}

Images of tissue sections were recorded with a CCD camera mounted on an inverted microscope. Approximately 10-15 representative areas were selected for each sample. A reference image was taken in a blank area on each slide outside the tissue. The illumination conditions were carefully adjusted so that the average light intensity of the reference image ( 8 bits after digitalization) remained between 210 and 220 . This condition was determined in a pilot study to avoid image saturation and interference with the estimation of light absorption.

In a tissue section labeled colorimetrically, the density of the targeted antigen is correlated with the density of pigment precipitated on the tissue through a chromogenic process. The density of pigment can be estimated by measuring the light absorption, $\mathrm{L}_{\mathrm{a}}$, of the tissue section, defined as

$$
\mathrm{L}_{\mathrm{a}}=\log _{10}\left(\mathrm{I}_{0} / \mathrm{I}\right)
$$

where $\mathrm{I}$ is the transmitted light intensity, and $\mathrm{I}_{0}$ is the incident light intensity. Light absorption is independent of illumination intensity. In addition, the light absorption of a mounted tissue section equals to the sum of the light absorptions of the glass slide, the tissue section, the mounting media and the cover slip. Since glass slide, coverslip and mounting media are almost identical within each slide, the light absorption of the tissue section alone can be calculated as

$$
\mathrm{L}_{\mathrm{a}}=\log _{10}\left(\mathrm{I}_{0}^{*} / \mathrm{I}^{*}\right)
$$

where $\mathrm{I}^{*}$ is the light intensity in the image containing the labeled tissue, and $\mathrm{I}_{0}^{*}$ is the intensity in a blank area of the slide without tissue. Light absorption was calculated for each pixel of the digitized images. For liver sinusoids, renal glomeruli and renal medulla, ICAM-1 label intensity was estimated by total light absorption divided by the area of selected tissues. For hepatic central veins and the vessels in heart and skeletal muscle, ICAM-1 label intensity was determined by total light absorption of the vessel cross-sections divided by the circumference of selected vessels. For renal glomerular capsules, ICAM-1 label intensity was estimated by maximum light absorption computed at three locations randomly selected along the capsule membrane.

In order to separate true primary antibody labeling from the background due to nonspecific labeling, the histogram of light absorption was computed for the representative images taken from the negative-control sections. The mean plus 3 standard deviations of the absorption calculated from the histogram was used as a threshold. Only the pixels with a light absorption above the threshold were considered to be 'labeled'. Image analysis was carried out with a custom computer program.

\section{ELISA for Soluble ICAM-1 in Plasma}

The plasma samples were stored at $-80^{\circ} \mathrm{C}$ and thawed at room temperature immediately before use. All plasma samples were analyzed at the same time using a Quantikine rat sICAM-1 immunoassay according to the manufacturer's instructions.

\section{Measurement of Elastase Activity}

The elastase activity of neutrophils was measured with a fluorogenic substrate, AAPV-methylcoumarin, (ex/em 370/460 nm). Blood smears of SHR and Wistar rat blood aliquots on glass slides after drying were incubated with $0.1 \mathrm{mM}$ substrate in buffer (Triz $0.05 \mathrm{M}$, THAM $0.5 \mathrm{M}, \mathrm{CaCl}_{2} 0.1 \mathrm{M}, \mathrm{pH} 7.5$ ) at $37^{\circ} \mathrm{C}$ for $60 \mathrm{~min}$. Fluorescence images were recorded and the fluorescence intensity of individual neutrophils was determined digitally.

The elastase activity of the plasma samples was determined with a synthetic substrate, SucAla ${ }_{3}$-PNA, using a colorimetric assay [31]. SucAla 3 -PNA $(0.3 \mathrm{mM})$ was dissolved in $100 \mathrm{mM} \mathrm{pH} 8.0$ Tris- $\mathrm{HCl}$ buffer with $0.32 \mathrm{mg} / \mathrm{ml} \mathrm{NaN}_{3}$ and $0.02 \%$ Tween-20. A volume of $145 \mu$ l SucAla 3 -PNA solution was mixed with either (a) $5 \mu \mathrm{l}$ plasma, (b) $5 \mu \mathrm{l}$ elastase solution $(0.5 \mathrm{mg} / \mathrm{ml})$ or (c) $5 \mu \mathrm{l}$ elastase solution plus $10 \mu \mathrm{l}$ plasma in a 96-well plate and incubated at $37^{\circ} \mathrm{C}$. For each sample, a blank was prepared by mixing the sample with Tris-HCl buffer. The light absorption at $405 \mathrm{~nm}$ was measured after incubation at room temperature for $10 \mathrm{~min}$. All samples were measured in duplicate.

\section{Cleavage of ICAM-1 by Proteases}

The cleavage of ICAM-1 by elastase, MMP-7 and MMP-9 was evaluated using fresh sections of inferior vena cava and internal jugular vein. Segments of vena cava and jugular veins from a Wistar rat were frozen in liquid nitrogen and sectioned with a cryostat. The tissue sections were brought to room temperature and rehydrated with PBS. Thereafter, the tissue sections were incubated with (1) PBS, (2) $0.5 \mathrm{mg} / \mathrm{ml}$ elastase, (3) $200 \mathrm{nM} \mathrm{MMP-7} \mathrm{or}$ (4) $200 \mathrm{nM}$ MMP- 9 at $37^{\circ} \mathrm{C}$ for $12 \mathrm{~h}$. After incubation, the sections were labeled with an anti-ICAM-1 antibody (1A29) and light absorption was quantified.

\section{Statistics}

Measurements are presented as means \pm standard deviation. Immunohistochemical labeling of ICAM-1 was carried out on paired tissue sections from SHRs and Wistar rats. Comparisons of mean labeling intensities between groups were carried out with a paired $t$ test. Other data were analyzed by a two-sample $t$ test. $\mathrm{p}<0.05$ was considered significant. 

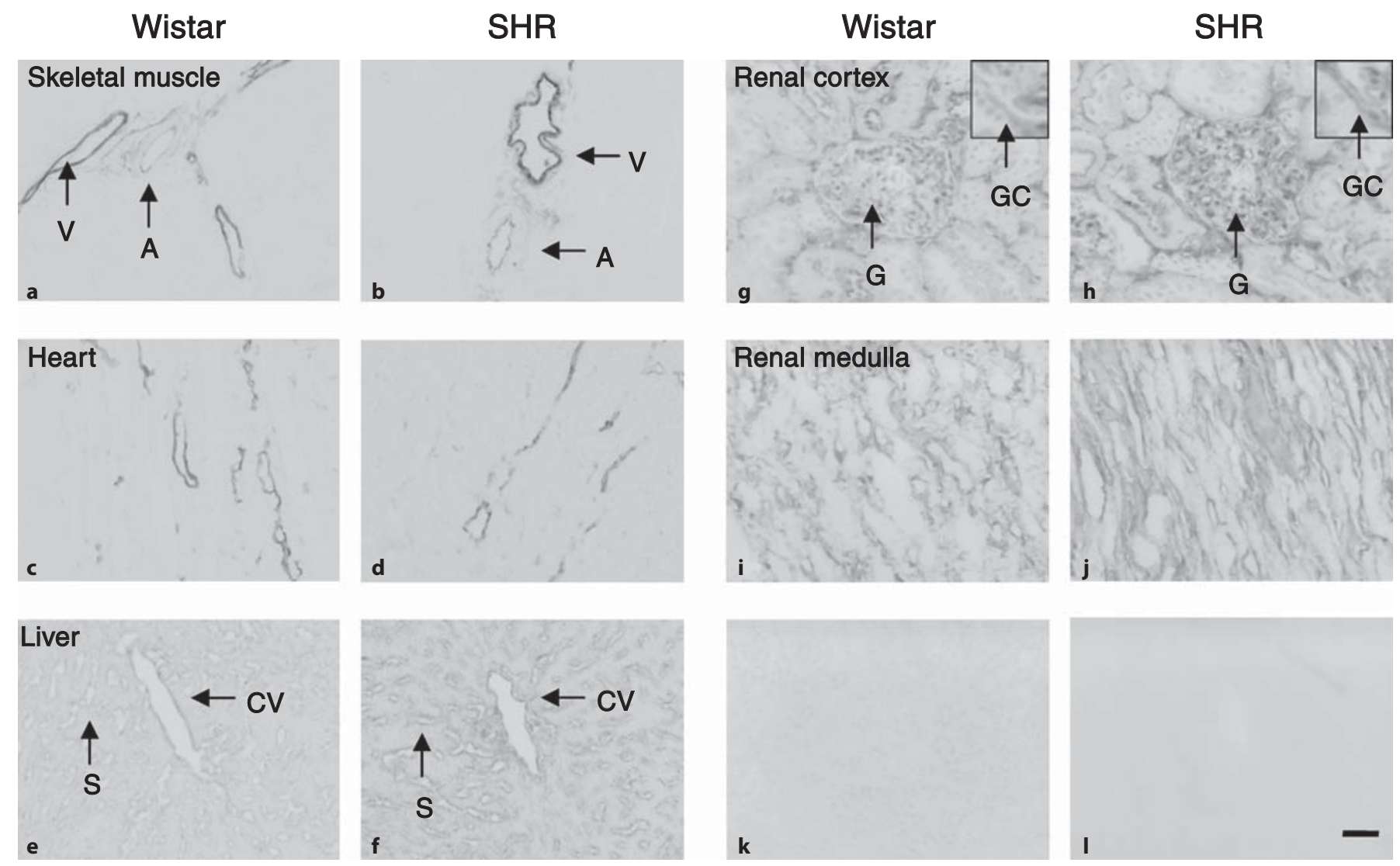

Fig. 1. Micrographs of selected tissue cryosections after labeling with an antibody against the extracellular domain of ICAM-1 (1A29). k, I are negative control sections of renal cortex and renal medulla, respectively, after replacement of primary antibody with control mouse IgG. Negative controls of other tissues have similar label intensity. A = Artery; V = vein; CV = liver central vein; $\mathrm{S}=$ liver sinusoid; $\mathrm{G}=$ renal glomeruli; $\mathrm{GC}=$ glomerular capsule. The scale bar represents $50 \mu \mathrm{m}$.

\section{Results}

SHRs had a significantly higher blood pressure compared to Wistar rats (table 1).

In all tissues examined, the SHRs and Wistar rats exhibited qualitatively similar ICAM-1 immunohistochemical label patterns for both intra- and extracellularly targeted antibodies (fig. 1, 2). In cardiac and skeletal muscle, the label for antibody against the extracellular domain of ICAM-1, 1A29, was observed primarily on the endothelium of veins and to a lesser extent on arteries (fig. 1a-d). In the liver, the extracellular domain of ICAM1 was found on the surface of central veins and sinusoids (fig. 1e, f). The renal structures, including glomeruli, glomerular capsules and medulla, were labeled more intensely for the extracellular domain of ICAM-1 compared to other tissues (fig. $1 \mathrm{~g}-\mathrm{j}$ ).
To evaluate the label density, we determined the light absorption for each tissue structure. Quantitative analysis with antibody $1 \mathrm{~A} 29$ indicated a significant increase in label density in liver sinusoids, central veins, renal glomeruli and the glomerular capsules of SHRs compared to Wistar rats (fig. 3a, b, 4a). In general, SHRs showed a trend towards a higher density of this immune label in other tissues as well, but differences between the two strains were not significant.

The extracellular domain of ICAM-1 on vascular endothelium may be subject to proteolytic cleavage. To distinguish between ICAM-1 fragments and intact ICAM1, the ICAM-1 labeling was repeated with antibody M-19 targeting the intracellular domain of ICAM-1 (fig. 2). M-19 labeling yielded different ICAM-1 distributions in the liver and kidney but not in the other organs we studied. In the liver, the M-19 label was found on the surface 

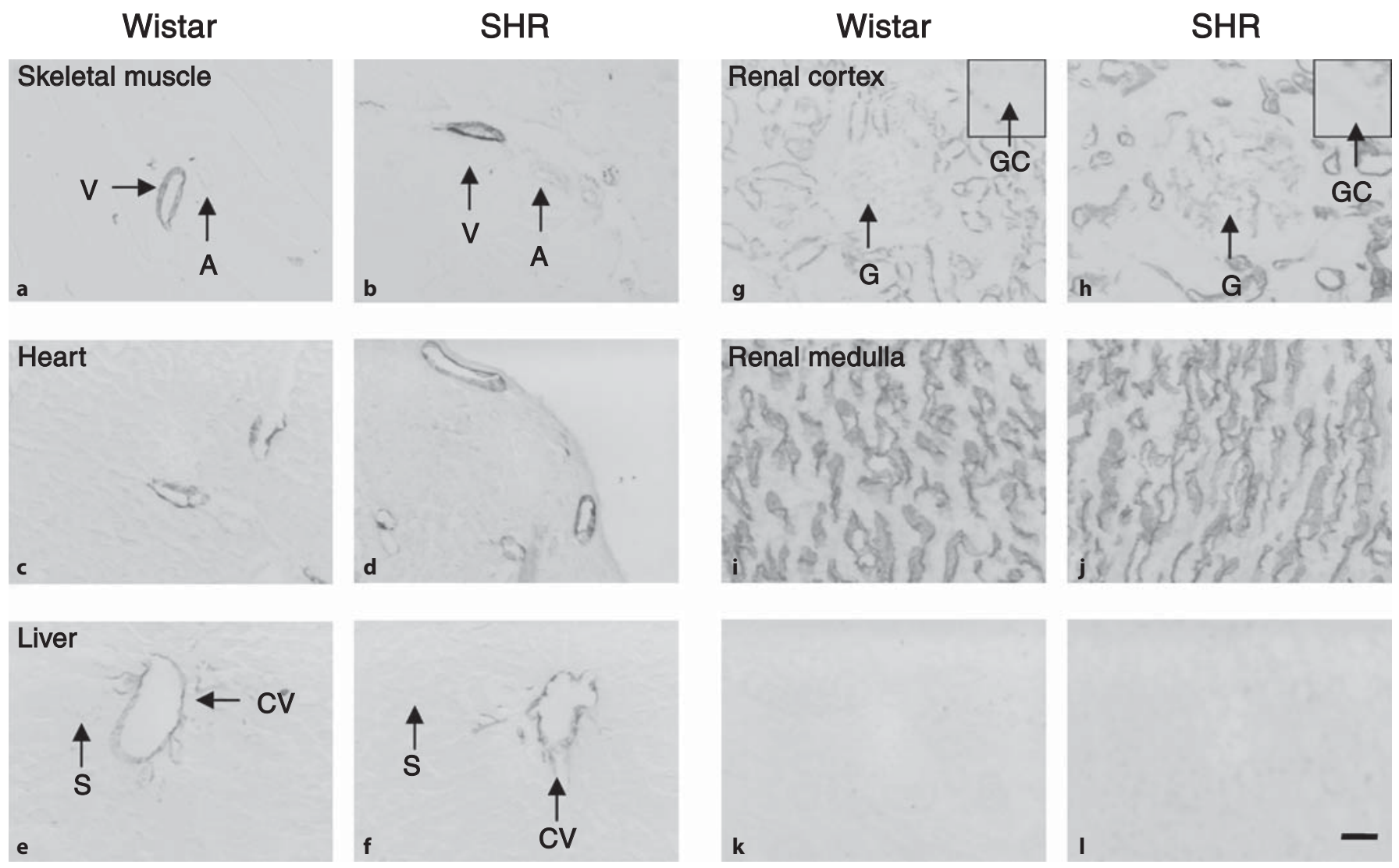

Fig. 2. Micrographs of selected tissue cryosections after labeling with an antibody against the intracellular domain of ICAM-1 (M-19). k, I are negative control sections of renal cortex and renal medulla, respectively, after replacement of primary antibody with control goat IgG. Negative controls of other tissues have similar label intensity. A = Artery; V = vein; CV = liver central vein; $\mathrm{S}=$ liver sinusoid; $\mathrm{G}=$ renal glomeruli; $\mathrm{GC}=$ glomerular capsule. The scale bar represents $50 \mu \mathrm{m}$.

of central veins but not in the surrounding sinusoids (fig. 2e, f). While the renal tubules and medulla remained intensely labeled by M-19, the M-19 label on the glomeruli, especially on the glomerular capsule, was considerably reduced (fig. 2g, h with insets). Therefore, the $1 \mathrm{~A} 29$ label in the liver sinusoids and the renal glomeruli may be largely due to accumulation of extracellular fragments of ICAM-1 from the systemic circulation. The M-19 label intensity in the SHR glomeruli and glomerular capsule was higher than in Wistar rats, as detected with the antibody against the extracellular domain (fig. 3c, 4b). In addition, the intracellular label intensity in the SHR renal medulla was higher than in Wistar rats (fig. 3c).

The concentration of soluble ICAM-1 in the plasma samples collected from the same group of animals, however, was lower in SHRs than in Wistar rats, which is not in line with findings in hypertensive patients (fig. 5). Note that the plasma level of soluble ICAM-1 in the rat is an order of magnitude lower than in the mouse and in humans [32]. It is unclear whether the low ICAM-1 level in rat plasma is due to rapid clearance of the protein fragments from the blood circulation, as seen in the liver sinusoids and glomerular capsule, or to multiple cleavage of ICAM-1 by proteases, which may prevent such fragments from detection by antibody binding.

The presence of ICAM-1 fragments in the liver sinusoids and in the renal glomeruli indicates that ICAM-1 cleavage occurs in both SHRs and Wistar rats. ICAM-1 can be cleaved by several proteases. To confirm ICAM-1 cleavage in situ, we incubated inferior vena cava and internal jugular vein sections with MMP-7, MMP-9 and elastase (fig. 6). Examination with antibody 1A29 showed that acute treatment with all three proteases significantly 
Fig. 3. Quantitative analysis of ICAM-1 label intensity. ICAM-1 label intensity on tissue sections labeled with the extracellular domain antibody 1A29 (a, b) and the intracellular domain antibody M-19 (c, d) is evaluated by light absorption, calculated as described in the Material and Methods section. The number of animals per group was 6 for renal cortex and medulla and 3 elsewhere. ${ }^{*} \mathrm{p}<0.05$ versus Wistar.
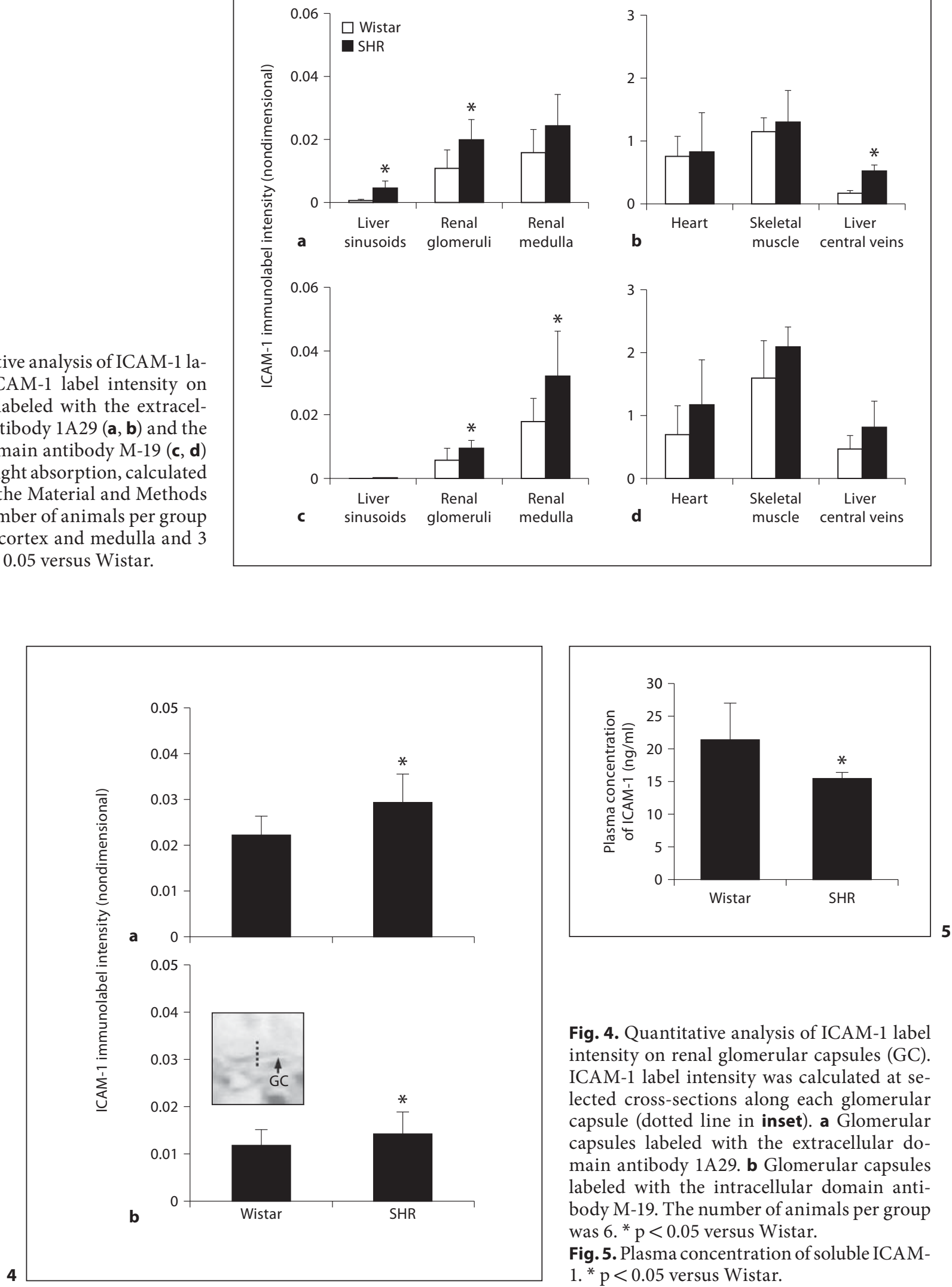

Fig. 4. Quantitative analysis of ICAM-1 label intensity on renal glomerular capsules (GC). ICAM-1 label intensity was calculated at selected cross-sections along each glomerular capsule (dotted line in inset). a Glomerular capsules labeled with the extracellular domain antibody 1A29. b Glomerular capsules labeled with the intracellular domain antibody M-19. The number of animals per group was $6 .{ }^{*} \mathrm{p}<0.05$ versus Wistar.

Fig. 5. Plasma concentration of soluble ICAM1. ${ }^{*} \mathrm{p}<0.05$ versus Wistar. 


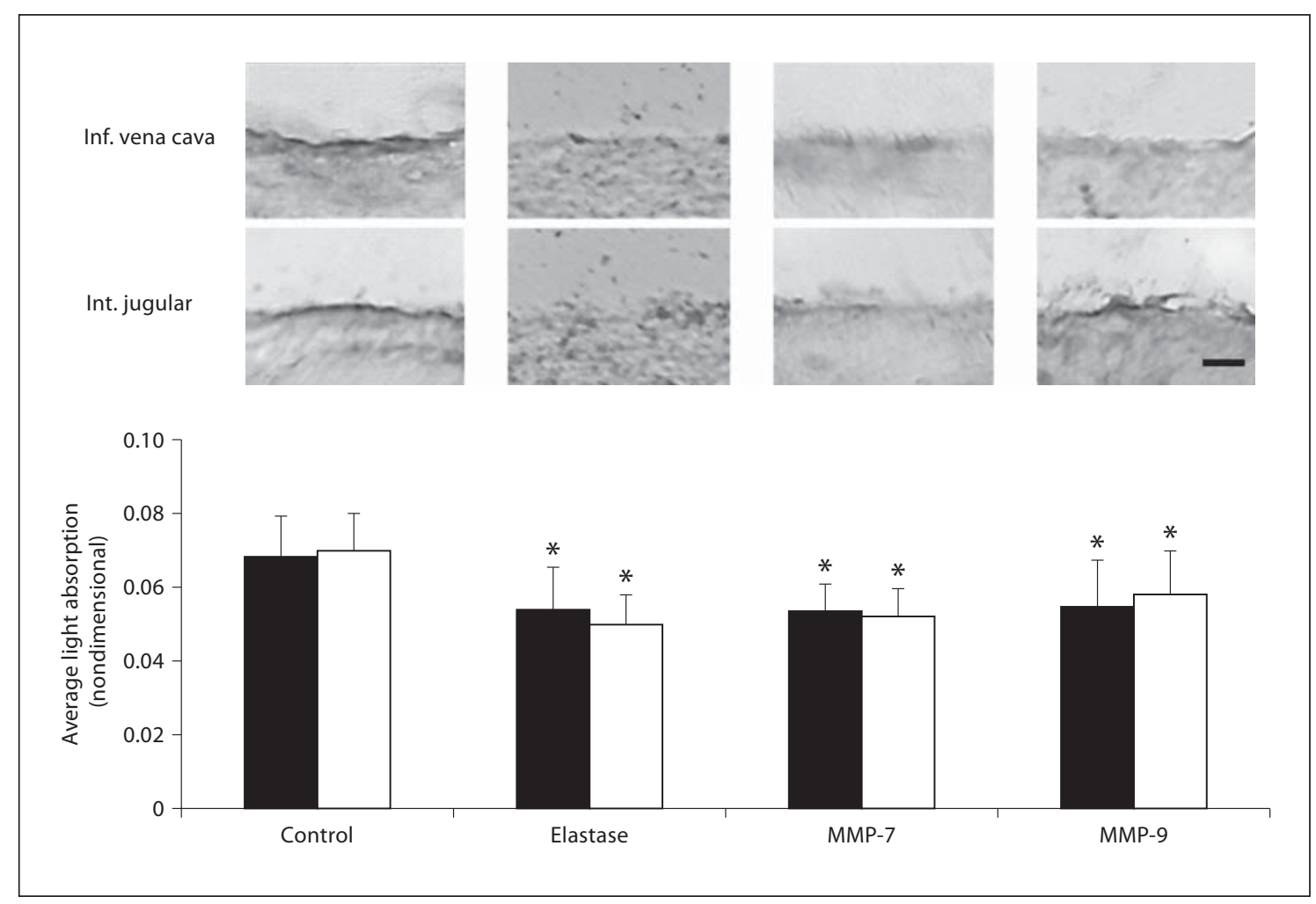

Fig. 6. Enzymatic cleavage of ICAM-1. Sections of inferior vena cava $(\square)$ and internal jugular vein $(\square)$ were incubated with PBS, elastase, MMP-7 and MMP-9. After incubation, the sections were stained with an antibody against the extracellular domain of ICAM-1 (1A29). Top: micrographs of tissue sections; bottom: quantitative analysis of ICAM-1 label intensity. ${ }^{*} \mathrm{p}<0.05$ versus control. The scale bar represents $10 \mu \mathrm{m}$.

reduced ICAM-1 level in the tissue sections, indicating that the extracellular domain of ICAM-1 on vascular endothelium was cleaved. SHRs display enhanced MMP activity in plasma and postcapillary venules, and plasma is also involved in the downregulation of CD18 in the SHR [29]. These results then suggest that the MMPs may also play a role in ICAM-1 distribution.

We further examined the elastase levels in the blood of SHRs and Wistar rats. The SHR has an increased number of circulating leukocytes and elevated levels of neutrophil degranulation [33, 34]. Neutrophil elastase is stored in the azurophil granules of neutrophils and released upon neutrophil degranulation. Incubation of the blood smear with a synthetic elastase substrate showed that there is higher elastase activity on the cell surface of SHR neutrophils compared with Wistar rats (fig. 7a). In contrast to plasma from hypertensive patients, elastase activity was not detectable in rat plasma samples (fig. 7b). However, plasma from both species was able to partially block the cleavage of $\mathrm{SucAla}_{3}$-PNA by porcine pancreatic elastase (fig. 7b). Interestingly, the inhibitory potential of SHR plasma was significantly reduced compared with that of Wistar rats.

\section{Discussion}

In this study, we compared the distribution of ICAM1 in SHRs and Wistar rats based on immunohistochemical labeling of tissue sections and plasma ELISA. With an antibody targeting the extracellular domain of ICAM-1 (1A29), there was no significant difference in ICAM-1 expression levels on heart and skeletal muscle vascular endothelium; this observation is in line with the results of Komatsu et al. [19], who evaluated ICAM-1 at the organ level by perfusion with radioisotope-labeled antibody 1A29. But we found a higher level of extracellular domain ICAM-1 in liver sinusoids, central veins, renal glomeruli and glomerular capsules of SHRs using antibody 1A29. In addition, using an antibody against the intracellular 


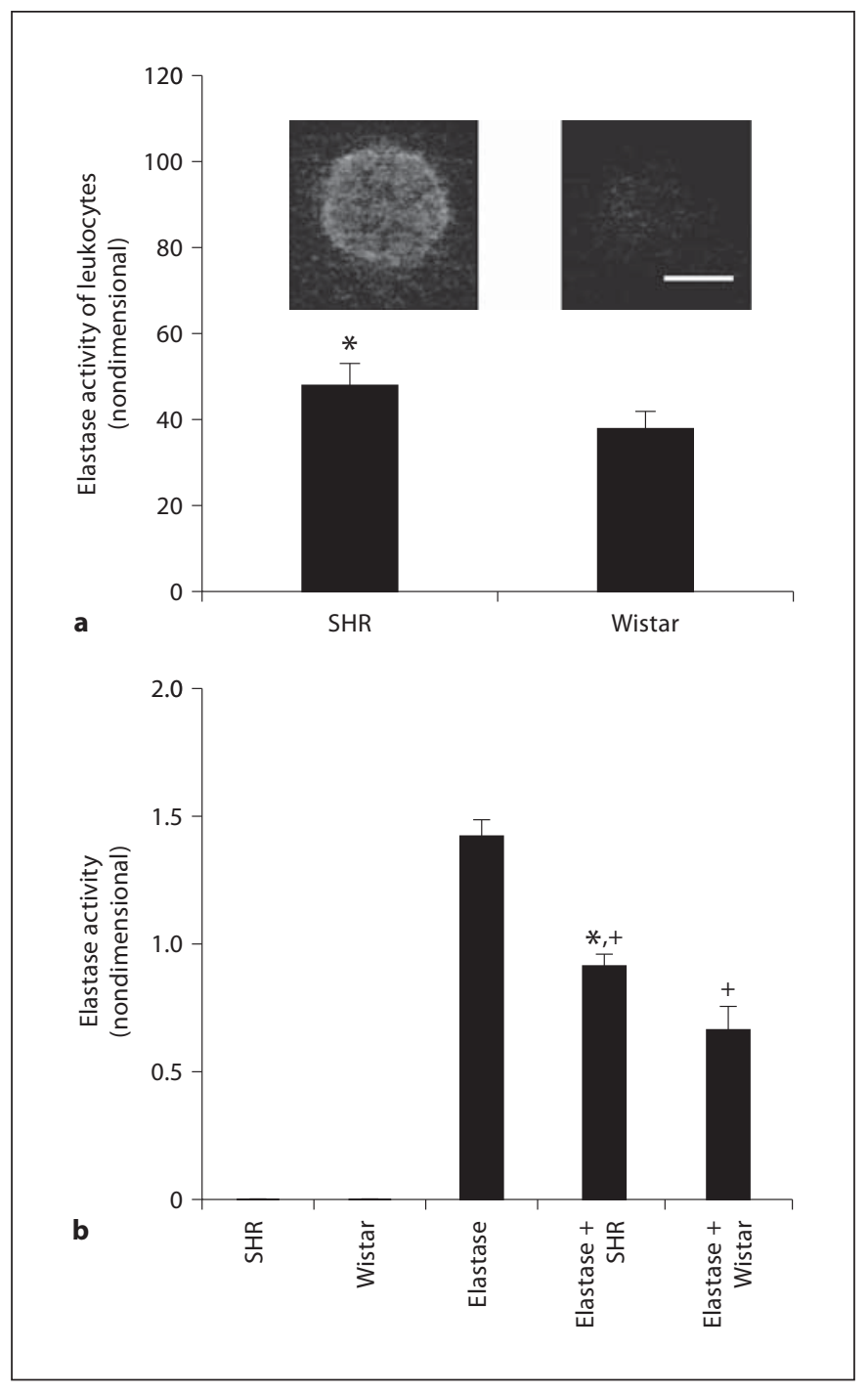

Fig. 7. Elastase activity of blood. a Elastase activity of neutrophils. Insets: fluorescence images of neutrophils after treatment with a fluorogenic elastase substrate. b Elastase activity of plasma. ${ }^{*} \mathrm{p}<$ 0.05 versus Wistar; ${ }^{+} \mathrm{p}<0.05$ versus control. The scale bar represents $5 \mu \mathrm{m}$.

domain (M-19), the label intensity was shown to be increased in renal glomeruli, glomerular capsules and medulla of SHRs. Comparison between 1A29 and M-19 labeling intensities suggests accumulation of ICAM-1 extracellular fragments in hepatic sinusoids and renal glomeruli.

Earlier studies based on several different hypertension models have shown that damage found in brain, heart, kidney and vessel wall is associated with increased interstitial infiltration of monocytes/macrophages and lym-

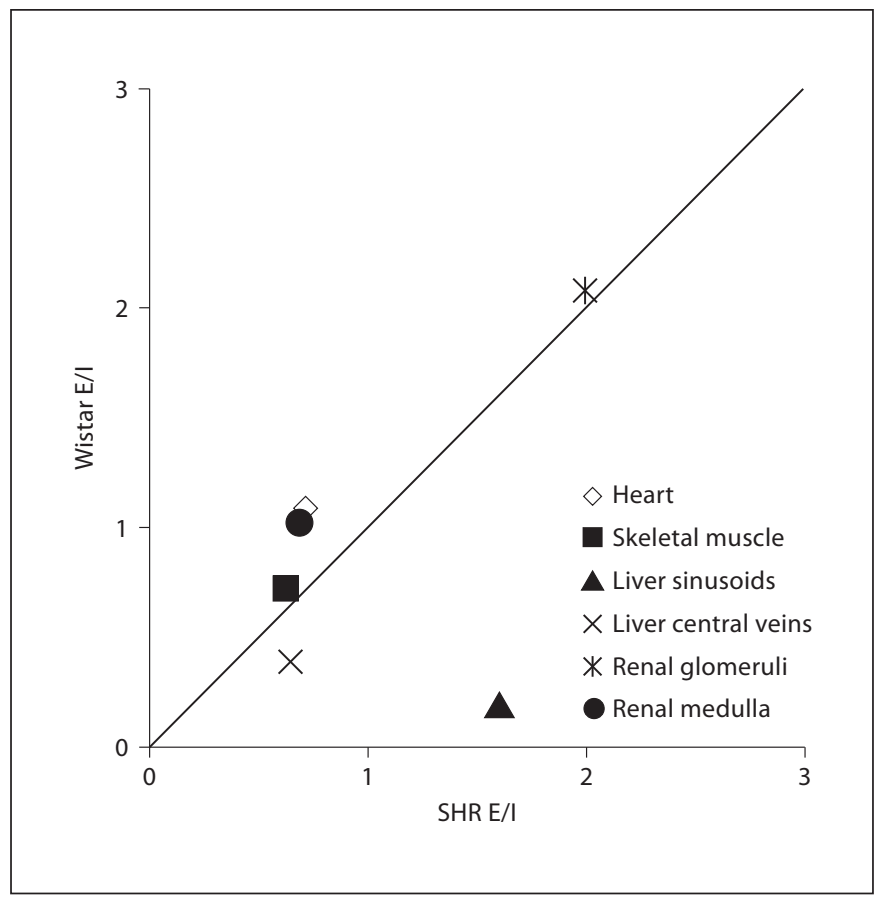

Fig. 8. Cross-correlation of the ratio between average extra- and intra-cellular label density of ICAM-1 (E/I) in different Wistar and SHR tissues. Each point represents the average E/I ratio (the label intensity of 1A29 divided by that of M-19) for one tissue of SHRs and Wistar rats. The line of identity represents equal labeling ratios in the two strains.

phocytes [1]. Leukocyte infiltration occurs preferentially at local 'hot spots' with a high level of adhesion molecules rather than an overall increase in whole organs. Our finding of increased levels of extra- and intracellular domain densities in several organs support the hypothesis that ICAM-1 may be important in facilitating increased leukocyte infiltration and, consequently, tissue damage in SHRs. In particular, elevated tubulointerstitial infiltration of lymphocytes and macrophages precedes the establishment of hypertension in SHRs [5]. Tubulointerstitial accumulation of lymphocytes and macrophages can lead to upregulation of renal oxidative stress and angiotensin II production, which subsequently affect salt reabsorption and pressure natriuresis [35]. Multiple pieces of evidence also showed that interventions that reduce renal immune infiltration can improve hypertension. In a salt hypertension model, overload proteinuria was associated with hypertension caused by renal infiltration of immune cells, and administration of an immunosuppressive drug, mycophenolate mofetil, prevented proteinuria-related interstitial nephritis [36]. Antihypertensive drugs such as 
amlodipine and hydralazine reduce leukocyte migration, which is accompanied by a decreased ICAM-1 expression $[15,16]$. Our data indicate that there is a higher level of intracellular domain of ICAM-1 in the SHR kidney, which provides additional evidence for an inflammatory state of renal tissue and may contribute to renal infiltration of immunocompetent cells. Furthermore, the extracellular fragments of ICAM-1 can form dimers in the plasma and bind to lymphocyte function-associated antigen type 1 (LFA-1) with high avidity $[37,38]$. There is a possibility that accumulated extracellular domain of ICAM-1 plays a role in renal leukocyte infiltration.

Although it has been shown that the extracellular domain of ICAM-1 can be cleaved by elastase, MMP-9 and cathepsin $\mathrm{G}$ in vitro, the extent to which proteolytic cleavage modulates the in vivo distribution of ICAM-1 is not well understood [23-25]. Plasma levels of soluble ICAM-1 are often increased in patients with acute or chronic inflammation. This increase may be due to either an overall increase in ICAM-1 expression or increased enzymatic activity associated with elevated leukocyte activation during inflammation. In contrast, we found that the level of soluble ICAM-1 in SHRs was lower than in Wistar rats. The SHR secretes proteins/peptides at higher levels [39], presumably due to higher permeability to large molecules, in spite of a reduced glomerular filtration rate [40]. This may contribute to increased accumulation of ICAM-1 fragments in glomerular capsules and a lower plasma level of ICAM-1 fragments in the SHR. This discrepancy may also be related to attachment of soluble ICAM-1 to its counterreceptor CD18 on circulating leukocytes [37, 38], or further enzymatic cleavage of the extracellular domain that results in undetectable fragments by the antibodies used in the ELISA assay. Nonetheless, the substantial amount of extracellular ICAM-1 fragments in the liver and kidney suggests that enzymatic cleavage may play an important role in regulating ICAM1 expression on vascular endothelium. With the current method, it is not possible to directly compare the level of intra- and extracellular domain due to the difference in the affinity of the two antibodies and the nature of the chromogenic labeling procedures. But comparing the ratio of the extra- to the intracellular labeling intensity permits assessment of the relative level of enzymatic cleavage in the two rat strains. As shown in figure 8, there is a trend for more cleavage in the heart, muscle and kidney of SHRs than in Wistar rats.

It is also important to note that ICAM-1 expression can be upregulated by several cytokines involved in hypertension, such as C-reactive protein, angiotensin II and oxygen free radicals. This increased expression compensates for the elevated enzymatic cleavage of ICAM-1 in SHRs. Furthermore, enzymatic cleavage may only occur in a proportion of ICAM-1 due to the accessibility and selectivity of the enzymes. For example, neutrophil elastase is stored in azurophilic granules and released upon neutrophil activation, a process that is enhanced in the SHR [33]. Most elastase, however, is retained on the surface of activated neutrophils and reaches a high local concentration [41]. As a result, the ICAM-1 molecules that actively bind to neutrophils are most likely subject to focal cleavage by neutrophil elastase. A similar situation is found in the case of CD18, which is cleaved by cathepsin B only in the activated state [42].

Several proteases have been reported to cleave ICAM1 molecules. The ones that probably act on vascular endothelium are MMPs and neutrophil elastase. We found that SHR plasma had enhanced MMP activities, which contributes to the reduction of an ICAM-1 receptor, CD18, and probably ICAM-1 itself, based on the current results $[28,29]$. In vitro, neutrophils isolated from SHRs induced damage to activated endothelial cells by elastase upon direct cellular contact while isolated medium has no effect [14]. In agreement with these observations, our results showed that elastase activity on the surface of neutrophils is increased in SHRs, while elastase activity in plasma is negligible. In addition, the plasma from healthy individuals can effectively inhibit elastase activity, presumably due to the presence of elastase inhibitors such as $\alpha_{1}$-antitrypsin [31]. The vascular endothelium in the microcirculation can be a potential target of elastase derived from activated circulating leukocytes since they are in close proximity when leukocytes pass through microvessels. The lack of plasma inhibition in SHRs, which have more activated circulating leukocytes than Wistar rats [33], may increase the enzymatic cleavage of surface molecules such as ICAM-1 and damage to vascular endothelium. Furthermore, other cell surface molecules are subject to enzymatic cleavage since these enzymes can degrade a broad spectrum of proteins [29]. Therefore, increased enzyme activity in the SHR may potentially reduce the responsiveness of the vascular network to agonist/antagonist signals by cleaving a variety of membrane receptors, as recently shown for the vascular endothelial growth factor receptor [28].

\section{Acknowledgement}

This study was supported by NIH grant HL 10881. 


\section{References}

$\checkmark 1$ Hilgers KF: Monocytes/macrophages in hypertension. J Hypertens 2002;20:593-596.

-2 Suematsu M, Suzuki H, Delano FA, SchmidSchönbein GW: The inflammatory aspect of the microcirculation in hypertension: oxidative stress, leukocytes/endothelial interaction, apoptosis. Microcirculation 2002;9: 259-276.

>3 Guzik TJ, Hoch NE, Brown KA, McCann LA, Rahman A, Dikalov S, Goronzy J, Weyand C, Harrison DG: Role of the T cell in the genesis of angiotensin II induced hypertension and vascular dysfunction. J Exp Med 2007;204:2449-2460.

4 Clozel M, Kuhn H, Hefti F, Baumgartner HR: Endothelial dysfunction and subendothelial monocyte macrophages in hypertension. Effect of angiotensin converting enzyme inhibition. Hypertension 1991;18:132141.

5 Rodriguez-Iturbe B, Quiroz Y, Ferrebuz A, Parra G, Vaziri ND: Evolution of renal interstitial inflammation and NF- $\mathrm{B}$ activation in spontaneously hypertensive rats. Am J Nephrol 2004;24:587-594.

-6 Springer TA: Traffic signals for lymphocyte recirculation and leukocyte emigration: The multistep paradigm. Cell 1994;76:301-314.

7 Suematsu M, Suzuki H, Tamatani T, Iigou Y, DeLano FA, Miyasaka M, Forrest MJ, Kannagi $\mathrm{R}$, Zweifach BW, Ishimura $\mathrm{Y}$, et al: Impairment of selectin-mediated leukocyte adhesion to venular endothelium in spontaneously hypertensive rats. J Clin Invest 1995; 96:2009-2016.

8 Suzuki H, Schmid-Schönbein GW, Suematsu M, DeLano FA, Forrest MJ, Miyasaka M, Zweifach BW: Impaired leukocyte-endothelial cell interaction in spontaneously hypertensive rats. Hypertension 1994;24:719-727.

-9 Arndt H, Smith CW, Granger DN: Leukocyte-endothelial cell adhesion in spontaneously hypertensive and normotensive rats. Hypertension 1993;21:667-673.

$\checkmark 10$ Mulivor AW, Lipowsky HH: Inhibition of glycan shedding and leukocyte-endothelial adhesion in postcapillary venules by suppression of matrix metalloprotease activity with doxycycline. Microcirculation 2009:110.

-11 Carman CV, Jun CD, Salas A, Springer TA: Endothelial cells proactively form microvilli-like membrane projections upon intercellular adhesion molecule 1 engagement of leukocyte LFA-1. J Immunol 2003;171:61356144.

-12 Entman ML, Youker K, Shoji T, Kukielka G, Shappell SB, Taylor AA, Smith CW: Neutrophil induced oxidative injury of cardiac myocytes. A compartmented system requiring CD11b/CD18-ICAM-1 adherence. J Clin Invest 1992;90:1335-1345.
13 Korthuis RJ, Anderson DC, Granger DN: Role of neutrophil-endothelial cell adhesion in inflammatory disorders. J Crit Care 1994; 9:47-71.

14 Ofosu-Appiah W, Sfeir G, Smith D, Richard T: Neutrophil-mediated damage to vascular endothelium in the spontaneously hypertensive rat. Clin Immunol Immunopathol 1997; 83:293-301.

15 Rodrigues SF, Dos Santos RA, de Oliveira MA, Rastelli VM, Nucci G, Tostes Rde C, Nigro $\mathrm{D}$, Carvalho $\mathrm{MH}$, Fortes ZB: Amlodipine reduces the antimigratory effect of diclofenac in spontaneously hypertensive rats. J Cardiovasc Pharmacol 2008;51:492-504.

16 Rodrigues SF, de Oliveira MA, dos Santos RA, Soares AG, de Cassia Tostes R, Carvalho $\mathrm{MH}$, Fortes ZB: Hydralazine reduces leukocyte migration through different mechanisms in spontaneously hypertensive and normotensive rats. Eur J Pharmacol 2008, 589:206-214.

17 Muller DN, Dechend R, Mervaala EM, Park JK, Schmidt F, Fiebeler A, Theuer J, Breu V, Ganten D, Haller H, Luft FC: NF-кB inhibition ameliorates angiotensin II-induced inflammatory damage in rats. Hypertension 2000;35:193-201.

18 Ponthieux A, Herbeth B, Droesch S, Haddy N, Lambert D, Visvikis S: Biological determinants of serum ICAM-1, E-selectin, P-selectin and 1-selectin levels in healthy subjects: The Stanislas study. Atherosclerosis 2004;172:299-308.

19 Komatsu S, Panes J, Russell JM, Anderson DC, Muzykantov VR, Miyasaka M, Granger DN: Effects of chronic arterial hypertension on constitutive and induced intercellular adhesion molecule-1 expression in vivo. $\mathrm{Hy}$ pertension 1997;29:683-689.

20 Iigo Y, Suematsu M, Higashida T, Oheda J, Matsumoto K, Wakabayashi Y, Ishimura Y, Miyasaka M, Takashi T: Constitutive expression of ICAM-1 in rat microvascular systems analyzed by laser confocal microscopy. Am J Physiol 1997;273:H138-H147.

21 Hlubocka Z, Umnerova V, Heller S, Peleska J, Jindra A, Jachymova M, Kvasnicka J, Horky K, Aschermann M: Circulating intercellular cell adhesion molecule-1, endothelin-1 and von Willebrand factor-markers of endothelial dysfunction in uncomplicated essential hypertension: the effect of treatment with ACE inhibitors. J Hum Hypertens 2002;16:557-562.

-22 Abe E, Matsubara K, Ochi H, Ito M, Oka K, Kameda K: Elevated levels of adhesion molecules derived from leukocytes and endothelial cells in patients with pregnancy-induced hypertension. Hypertens Pregnancy 2003; 22:31-43.
23 Robledo O, Papaioannou A, Ochietti B, Beauchemin C, Legault D, Cantin A, King PD, Daniel C, Alakhov VY, Potworowski EF, St-Pierre Y: ICAM-1 isoforms: specific activity and sensitivity to cleavage by leukocyte elastase and cathepsin G. Eur J Immunol 2003;33:1351-1360.

24 Fiore E, Fusco C, Romero P, Stamenkovic I: Matrix metalloproteinase 9 (MMP-9/gelatinase B) proteolytically cleaves ICAM-1 and participates in tumor cell resistance to natural killer cell-mediated cytotoxicity. Oncogene 2002;21:5213-5223.

25 Champagne B, Tremblay P, Cantin A, St Pierre Y: Proteolytic cleavage of ICAM-1 by human neutrophil elastase. J Immunol 1998; 161:6398-6405.

26 van Den Engel NK, Heidenthal E, Vinke A, Kolb H, Martin S: Circulating forms of intercellular adhesion molecule (ICAM)-1 in mice lacking membranous ICAM-1. Blood 2000;95:1350-1355.

27 Yasmin, Wallace S, McEniery CM, Dakham Z, Pusalkar P, Maki-Petaja K, Ashby MJ, Cockcroft JR, Wilkinson IB: Matrix metalloproteinase-9 (MMP-9), MMP-2, and serum elastase activity are associated with systolic hypertension and arterial stiffness. Arterioscler Thromb Vasc Biol 2005;25:372378.

28 Tran ED, Delano FA, Schmid-Schönbein GW: Enhanced matrix metalloproteinase activity in the spontaneously hypertensive rat: VEGFR-2 cleavage, endothelial apoptosis, and capillary rarefaction. J Vasc Res 2010;47: 423-431.

29 DeLano FA, Schmid-Schönbein GW: Proteinase activity and receptor cleavage: mechanism for insulin resistance in the spontaneously hypertensive rat. Hypertension 2008; 52:415-423.

30 Yang D, Zhang Y, Nguyen HG, Koupenova M, Chauhan AK, Makitalo M, Jones MR, St Hilaire C, Seldin DC, Toselli P, Lamperti E, Schreiber BM, Gavras H, Wagner DD, Ravid $\mathrm{K}$ : The $\mathrm{A} 2 \mathrm{~B}$ adenosine receptor protects against inflammation and excessive vascular adhesion. J Clin Invest 2006;116:1913-1923.

31 Mathrubutham M, Rao SK, Shah NR, Cohen JR: Plasma elastase activity inhibition: a microassay. Clin Chem 1998;44:664-666.

-32 Heidenreich F, Arendt G, Jander S, Jablonowski H, Stoll G: Serum and cerebrospinal fluid levels of soluble intercellular adhesion molecule 1 (sICAM-1) in patients with HIV-1 associated neurological diseases. J Neuroimmunol 1994;52:117-126.

>33 Schmid-Schönbein GW, Seiffge D, DeLano FA, Shen K, Zweifach BW: Leukocyte counts and activation in spontaneously hypertensive and normotensive rats. Hypertension 1991;17:323-330. 
-34 Shen K, Sung KL, Whittemore DE, DeLano FA, Zweifach BW, Schmid-Schönbein GW: Properties of circulating leukocytes in spontaneously hypertensive rats. Biochem Cell Biol 1995;73:491-500.

-35 Quiroz Y, Pons H, Gordon KL, Rincon J, Chavez M, Parra G, Herrera-Acosta J, Gomez-Garre D, Largo R, Egido J, Johnson RJ, Rodriguez-Iturbe B: Mycophenolate mofetil prevents salt-sensitive hypertension resulting from nitric oxide synthesis inhibition. Am J Physiol Renal Physiol 2001;281:F38F47.
36 Alvarez V, Quiroz Y, Nava M, Pons H, Rodriguez-Iturbe B: Overload proteinuria is followed by salt-sensitive hypertension caused by renal infiltration of immune cells. Am Physiol Renal Physiol 2002;283:F1132F1141.

37 Miller J, Knorr R, Ferrone M, Houdei R, Carron $\mathrm{CP}$, Dustin ML: Intercellular adhesion molecule-1 dimerization and its consequences for adhesion mediated by lymphocyte function associated-1. J Exp Med 1995; 182:1231-1241.

-38 Barnett CC Jr, Moore EE, Moore FA, Carl VS, Biffl WL: Soluble icam-1 (sICAM-1) provokes PMN elastase release. J Surg Res 1996; 63:6-10.

$>39$ Feld LG, Van Liew JB, Galaske RG, Boylan JW: Selectivity of renal injury and proteinuria in the spontaneously hypertensive rat. Kidney Int 1977;12:332-343.
40 Uyehara CF, Gellai M: Impairment of renal function precedes establishment of hypertension in spontaneously hypertensive rats. Am J Physiol 1993;265:R943-R950.

41 Owen CA, Campbell MA, Sannes PL, Boukedes SS, Campbell EJ: Cell surfacebound elastase and cathepsin $\mathrm{G}$ on human neutrophils: a novel, non-oxidative mechanism by which neutrophils focus and preserve catalytic activity of serine proteinases. J Cell Biol 1995;131:775-789.

42 Shin HY, Simon SI, Schmid-Schönbein GW: Fluid shear-induced activation and cleavage of CD18 during pseudopod retraction by human neutrophils. J Cell Physiol 2008;214: 528-536. 